\title{
Efficacy and adherence of non-invasive ventilation treatment in children with Down syndrome
}

\author{
Lauren MacDonagh ${ }^{1}$, Lisa Farrell ${ }^{2}$, Ruth O'Reilly ${ }^{2}$, Paul McNally ${ }^{3}$, Sheila Javadpour ${ }^{4}$, and \\ Des $\operatorname{Cox}^{2}$ \\ ${ }^{1}$ University College Dublin School of Medicine \\ ${ }^{2}$ Our Lady's Children's Hospital, Crumlin \\ ${ }^{3}$ Our Lady's Children's Hospital \\ ${ }^{4}$ Our Lady's Children's Hospital Crumlin
}

September 28, 2020

\begin{abstract}
Objective: Children with Down syndrome (DS) have an increased prevalence of obstructive sleep apnoea (OSA). Non-invasive ventilation (NIV) is a common modality of OSA treatment in this cohort. This study aimed to measure adherence and efficiency of NIV delivery in children with DS. Study design: This was a retrospective cohort study involving 106 children with confirmed OSA and home NIV with downloadable data capacity. Children were divided into DS ( $\mathrm{n}=44)$ and non-DS cohorts (n=62). Adherence, clinical outcomes apnoea-hypopnoea index (AHI), positive airway pressure delivery and leakage were recorded and compared between DS and non-DS cohorts and within the DS cohort based on past surgical history. Results: Significantly greater NIV usage was observed in the DS cohort, they showed more consistent use with an increased percentage of days used relative to their non-DS counterparts $(78.95 \pm 2.26$ versus $72.11 \pm 2.14, \mathrm{p}=0.031)$. However, despite greater usage, poorer clinical outcomes in the form of increased AHI ( $\mathrm{p}=0.0493)$ was observed in the DS cohort, where significantly greater leakage was also shown $41.00 \pm 1.61 \mathrm{~L} / \mathrm{min}$ versus $36.52 \pm 1.18 \mathrm{~L} / \mathrm{min}(\mathrm{p}=0.022)$. Twenty children with $\mathrm{DS}$ had prior cardiac surgery; compliance across all parameters was significantly reduced relative to those without. Conclusions: These data confirm that satisfactory NIV adherence is achievable in children with DS. However, we have identified excessive system leak at the machine-patient interface as a factor, which could undermine NIV efficacy in children with DS.
\end{abstract}

\section{Introduction}

Down syndrome (DS), or Trisomy 21 is the most common chromosomal disorder observed worldwide in live-born children ${ }^{1}$. Occurrence rates vary widely globally, however, the rate of DS correlates with rising maternal age, which has been steadily increasing since the 1970's ${ }^{2}$. Reported DS birth rates range between 13.6-22.0/10,000 live births ${ }^{2,3}$. In Ireland, the most recent figures demonstrate that 1 in 444 live-born babies have DS ${ }^{2}$.

DS is characterised by a distinctive collection of phenotypic features in combination with developmental delay and intellectual impairment ${ }^{1}$. The phenotypic characteristics of DS are predominated by altered craniofacial anatomy, where mid-facial and mandibular hypoplasia, relative macroglossia, a posteriorly placed tongue, hypotonia, as well as, lymphoid, particularly adenotonsillar, hypertrophy culminate in a small and narrow upper airway ${ }^{4}$. The aforementioned phenotypic features in combination with the increased prevalence of congenital airway defects, such as laryngomalacia, tracheobronchomalacia, tracheal compression and subglottic stenosis predispose children with DS to obstructive sleep apnoea (OSA) ${ }^{5,6}$. The reported prevalence of OSA in the DS paediatric population ranges from 30-66.4\%, compared with $0.7-2 \%$ in the general paediatric population 7,8 . Furthermore, OSA is more severe within the DS population, particularly in the 
younger age groups ${ }^{7}$. There are numerous co-morbidities associated with DS which have been shown to be responsible, at least in part, for the severity of OSA within the DS population, including a predisposition to upper respiratory tract infections, hypothyroidism, gastro-oesophageal reflux (GORD) and obesity ${ }^{9-13}$. Within DS there is a propensity for congenital cardiac defects to affect the respiratory system, and vice versa, in which respiratory disease, including OSA, affects the heart and the pulmonary vasculature ${ }^{14}$. OSA-induced recurrent hypoxaemia has been shown to alter pulmonary vessel resistance leading the development, persistence and recurrence of pulmonary hypertension, right-sided heart failure and cor pulmonale ${ }^{14-16}$. Sleep disordered breathing has also been linked to enhanced sympathetic drive as well as worse left ventricular diastolic function ${ }^{17}$.

The management of OSA within the DS population involves a multidisciplinary approach. Upper airway surgery remains the preferred initial management for suitable candidates in moderate to severe OSA ${ }^{18}$. However, surgery is not always effective and residual OSA post-adenotonsillectomy has been reported to be as high as $70 \%{ }^{19-22}$. In such children, non-invasive ventilation (NIV) in the form of positive airway pressure, whether that be as continuous positive airway pressure (CPAP) or bi-level positive airway pressure (BiPAP), is the mainstay of OSA treatment ${ }^{23}$.

Effective management of OSA within the DS population is of the utmost importance. Poor OSA management has been associated with worsening cardiovascular issues, such as pulmonary hypertension, cardiovascular disease in adulthood, culminating in increased morbidity and mortality ${ }^{24-26}$. Moreover, the treatment of upper airway obstruction has been shown to reverse pulmonary hypertension ${ }^{26}$. The DS cohort has also been shown to be more susceptible to the negative impacts of sleep disturbances on neurocognitive and behavioural problems ${ }^{27}$.

There are conflicting results within the current body of literature of the efficacy and adherence to domiciliary NIV within the paediatric DS population. Previous studies have suggested poor tolerance and compliance to NIV within this cohort ${ }^{23}$. In this study we hypothesised that poor adherence is a key issue undermining the effectiveness of NIV in the DS population. The aim of this study was to assess adherence over time, as well as, the efficiency of NIV delivery to a paediatric DS cohort.

\section{Methods}

\subsection{Study Design}

A retrospective cohort study was conducted in Children's Health Ireland, Crumlin, the largest tertiary paediatric centre in Ireland. Children with DS aged 0-18 years and a confirmed diagnosis of OSA by polysomnography (PSG) who were commenced on NIV treatment were included in the study. Data from non-DS patients on NIV therapy attending our service were also collected for comparison. This study was approved by the hospital ethics committee.

Children attending our NIV services were identified via the downloadable data cloud platform (Fisher \& Paykel, Auckland, NZ) data were available from March 2014 to August 2019. A total of 106 children met inclusion criteria.

\subsection{Patient characteristics}

Age, sex, past medical and surgical history were noted. Based upon medical records, patients were divided into DS and non-DS cohorts. Within the DS cohort, any co-morbidity associated with DS was recorded. In particular, past cardiothoracic surgical history was noted, as was a history of upper airways surgery. Within the non-DS cohort the diagnosis of OSA or a syndrome or genetic alteration associated with sleep disordered breathing was recorded. The cohorts were divided into three age groups; age group one (0-5years), age group two (5-10years) and age group three (>10years).

\subsection{Polysomnography and NIV domiciliary data}

Polysomnography (PSG) was performed on all children overnight in the hospital sleep laboratory using the Philips Respironics Alice $6^{\mathrm{TM}}$ PSG system (Koninklijke Philips N.V., Netherlands) with Transcutaneous 
Capnography Radiometer TOSCA $500^{\mathrm{TM}}$ analyser and scored with the aid of Philips Respironics G3 ${ }^{\mathrm{TM}}$ software according to the American Academy of Sleep Medicine (AASM) paediatric criteria ${ }^{28,29}$.

NIV therapy was prescribed by a treating paediatric respiratory consultant, based on the PSG findings and symptomatology. NIV therapy was commenced using a previously prescribed pathway with the support of an NIV clinical nurse specialist ${ }^{30}$.

Domiciliary NIV data was collected via secure memory cards and modem technology. Only children with downloadable data available for at least one year were included in the analysis. Data recorded in this study included parameters relating to usage, function and clinical outcomes.

The usage parameters included pressure $\left(\mathrm{cmH}_{2} \mathrm{O}\right)$, humidity, percent days used, percentage days used $>4 \mathrm{hrs}$ and average use per night. Functional measures include system leak $(\mathrm{L} / \mathrm{min})$ and percentage of time with excess leak (\%). Clinical outcomes were measured using apnoea-hypopnoea index (AHI).

\subsection{Statistical analysis}

All data were analysed using GraphPad Prism ${ }^{\mathrm{TM}}$ software. Normality testing was performed on all data using the Kolmogorov-Smirnov test. Statistical comparison of two means was performed using an unpaired two-tailed Student's $t$-test should the data meet normality. A nonparametric Mann-Whitney U test was used if it did not. Data are presented as mean \pm standard error of the mean (SEM). A $p$-value [?] 0.05 was considered statistically significant.

\section{Results:}

\subsection{Study population}

Data was available for analysis on 106 patients, of which 44 were in the DS group and 62 were in the non-DS group (Table 1). Within both groups $55 \%$ were female. There was no significant difference in overall mean age between the DS and non-DS cohorts, $4.92+-0.68$ years and $5.54+-0.49$ years $(\mathrm{p}=0.449)$, respectively. However, further division of the cohorts into three age groups revealed a significant difference between the youngest age groups. The DS cohort within age group one $(n=22)$ were initiated on NIV significantly earlier than those in the non-DS cohort $(n=29), 0.98+-0.26$ years versus $2.19+-0.34$ years, $\mathrm{p}=0.011$.

A cardiac disorder was the most common co-morbidity associated with DS observed within this cohort (77\%). Twenty patients (45\%) of the cohort had previous cardiac surgery. Twelve children (27\%) in the cohort had a history of a congenital airway abnormality and $16(36 \%)$ had a past history of ENT surgery.

The most common diagnosis for which NIV treatment was initiated in the non-DS cohort was OSA; twentyfour children (36\%) had a primary diagnosis of OSA.

\subsection{NIV adherence and effect}

Comparisons between the DS and non-DS cohorts were made across a number of usage, functional and clinic outcome parameters (Figure 1). No significant difference was observed between cohorts in pressure or humidity (data not shown).

A significant difference was observed within the usage parameters between cohorts. The DS group showed significantly greater use compared with their non-DS equivalent, $78.95+-2.26 \%$ versus $72.11+-2.14 \%$ of all days used within one year $(\mathrm{p}=0.032)$. However, of the days used there was no significant difference in adequate usage on those nights, adequacy defined as greater than four hours. Both cohorts exhibited $>50 \%$ of nights with usage greater than four hours, $54.30+-2.98 \%$ and $59.61+-2.31 \%$, in the DS and non-DS cohorts, respectively. This data is reinforced by the parameter of average use per night, $4.85+-0.27$ hours within the DS cohort versus $5.07+-0.23$ hours in the non-DS group where again, no significant difference was observed between cohorts $(\mathrm{p}=0.543)$.

NIV efficacy data from home NIV devices was analysed. Those within the DS cohort had a significantly higher AHI measurement $(5.72+-0.47)$ relative to their non-DS counterparts $(4.42+-0.27), \mathrm{p}=0.049$. There 
was significantly greater system leak $(41.00+-1.61 \mathrm{~L} / \mathrm{min}$ versus $36.52+-1.18 \mathrm{~L} / \mathrm{min}, \mathrm{p}=0.022)$, as well as, percent time at excess leak $(21.02+-2.10$ versus $16.14+-1.29, \mathrm{p}=0.038)$ of the NIV system in the DS cohort compared with non-DS, respectively.

\subsection{NIV usage and effect by age}

Domiciliary data was compared across age groups, as well as, between and within cohorts (Figure 2). There was an increase in the percentage days used in the DS age group two relative to their non-DS comparators. Significantly increased usage was observed in the eldest DS age group relative to its corresponding age group one. There were no statistical differences observed in the usage parameter of percent days used $>4$ hours, between DS and non-DS cohorts in any age group. However, greater use was observed in the non-DS eldest cohort relative to its corresponding younger age groups. Similar trends were observed in the parameter of average use per night.

The clinical outcome of AHI was significantly higher in the youngest age group within the DS cohort relative to the non-DS, an AHI of 6.38 +- 0.74 was observed in the youngest DS age group compared with 4.74 +0.40 in the age-matched non-DS group, with $\mathrm{p}=0.041$.

Within the eldest two age bands, there was significantly greater system leak in the DS cohort relative to their age-matched counterparts, $45.34+-3.39 \mathrm{~L} / \mathrm{min}$ versus $34.88+-1.72 \mathrm{~L} / \mathrm{min}(\mathrm{p}=0.003)$ in age group two and $42.88+-3.60 \mathrm{~L} / \mathrm{min}$ versus $31.86+-2.56 \mathrm{~L} / \mathrm{min}(\mathrm{p}=0.013)$ in those aged greater than 10 years. The non-DS cohort showed a significant decrease in system leak as age increased; this trend was not mirrored in the DS group. A similar trend was also observed in the functional parameter of percentage of time at excess leak. Comparison across the 5-10 year age group shows a mean time at excess leak of $22.41 \%$ in those with DS and $14.01 \%$ in the non-DS group, with $\mathrm{p}=0.023$. Similarly, in those aged greater than 10 years old greater time at leakage was observed in the DS cohort, $23.78 \%$ compared to $11.86 \%, \mathrm{p}=0.024$.

\subsection{Upper airway surgery}

The DS cohort was divided into those with a past history of upper airway surgery, either adenotonsillectomy or tonsillectomy with subsequent residual OSA requiring NIV and those that had no past upper airway surgical history (Figure 3).

There were no significant differences observed in the usage parameters of percentage of days used and of days used $>4 \mathrm{hrs}$ between those who had past surgical intervention relative to those without. Both groups showed $>4 \mathrm{hrs}$ of use in over $50 \%$ of nights, $52.44+-5.17 \%$ in the surgical group and $59.35+-3.53 \%$ in those without. Conversely, the non-surgical group showed significantly greater average use per night of $5.31+-$ 0.32 hours/night compared to $4.14+-0.48$ hours/night in the surgical cohort, $\mathrm{p}=0.035$.

No statistical significance was observed in AHI measurements between the surgical and non-surgical cohorts, $6.23+-0.99$ and $5.41+-0.52$, respectively.

Increased leakage was observed within the surgical cohort. Mean system leakage of $47.53 \mathrm{~L} / \mathrm{min}$ was recorded in the surgical group compared $36.74 \mathrm{~L} / \mathrm{min}$ in the non-surgical group $(\mathrm{p}=0.001)$. Those within the surgical cohort also displayed significantly greater time at excess leak $(27.63+-4.07 \%)$ compared to their surgical naive comparators $(15.62+-1.90 \%), \mathrm{p}=0.003$.

\subsection{Cardiac surgery}

Seventy-seven percent of children with DS included in the analysis had a history of congenital cardiac disease and forty-five percent of the cohort $(\mathrm{n}=20)$ had previous cardiac surgery. NIV data were compared between those with a past history of cardiac surgery prior to NIV initiation and those without (Figure 4).

Those with a history of cardiac surgery showed significantly less NIV use, across all parameters. In terms of percentage of days used, those with prior cardiac surgery showed $71.18+-3.73 \%$ days used compared to $85.04+-2.68 \%$ in their non-surgical counterparts $(\mathrm{p}<0.001)$. As well as increased days used, the non-surgical cohort showed greater use per night, with a significantly greater percentage of days used $>4 \mathrm{hrs}(62.07+-$ 
$3.72 \%$ versus $49.83+-4.56, \mathrm{p}=0.037)$ and increased average use per night $(5.59+-0.34 \mathrm{hrs}$ versus $4.47+-$ $0.40 \mathrm{hrs}, \mathrm{p}=0.036$ ).

Significantly greater AHI was observed in the cardiothoracic surgical cohort relative to their counterparts, $6.86+-0.93$ and $4.75+-0.42(\mathrm{p}=0.031)$, respectively. However, despite poorer use the surgical cohort showed significantly greater system efficacy in the form of reduced system leak $(p=0.046)$ and percentage of time at excess leak $(\mathrm{p}=0.004)$ relative to their non-surgical comparators.

\section{Discussion}

To the best of our knowledge, this is the first paper to examine NIV adherence and efficacy in children with DS with a control population. Our study demonstrates that satisfactory NIV adherence and efficacy rates are achievable in children with DS compared with a non-DS cohort. Overall, acceptable leakage levels and NIV efficacy were demonstrated across different age groups and cohorts in our study. Children with DS with known congenital cardiac disease who underwent cardiac surgery had decreased NIV adherence when compared with children who had not undergone cardiac surgery.

There are conflicting reports regarding NIV adherence in children with DS throughout the literature. Previous studies have shown that poor compliance and refusal are significantly more common in children with DS due to behavioural and cognitive impairment ${ }^{31}$. We collected adherence data for twelve months in both DS and non-DS cohorts and conversely to previous studies, we have shown that children with DS showed increased usage relative to their non-DS counterparts, with the DS cohort using NIV on more than $75 \%$ of nights, with $>4 \mathrm{hrs}$ use on more than $50 \%$ of nights. These data are in keeping with that observed by Dudoignon et al, where good compliance was observed in the majority of children with DS ${ }^{20}$. Similarly, Trucco et al showed satisfactory NIV adherence within a paediatric DS population, with particular emphasis being placed on the initiation period as a key factor in overall and enduring compliance ${ }^{23}$. The importance of early optimisation and compliance is a sentiment echoed in the literature, Mansell et al have highlighted the important role of early domiciliary ventilator downloads to identify modifiable risk factors, such as adherence, as part of a treatment protocol to optimise and personalise NIV treatment ${ }^{32}$.

Having established satisfactory compliance within our DS paediatric cohort, we next looked at another key factor of therapeutic success, effective NIV delivery and system leakage. To date, there have been no studies investigating NIV leakage in a paediatric population; furthermore the level of clinical "tolerabilty" of leaks is not well established in home NIV ${ }^{33}$. Despite this, it is widely known that leakage will significantly effect NIV performance ${ }^{34}$. In this study we identified that there was significantly greater leak in the DS cohort relative to their non-DS counterparts and this is particularly evident in those aged $>5 \mathrm{yrs}$.

Upper airway surgery is often the first-line treatment of OSA in children with DS ${ }^{18}$. Although significant clinical improvements are seen post-adenotonsillectomy, the prevalence of residual OSA is reported to be between $47 \%$ and $70 \%^{22,35}$. In this study there was no difference in NIV adherence in those with residual OSA relative to those with no history of upper airway surgery. Furthermore, leak and time at excess leak was more pronounced in those with DS that displayed residual OSA post ENT surgery. This finding may have been due to craniofacial abnormalities which are well described in children with DS. Reducing leak at the patient-ventilator interface is important, not only to further promote comfort and compliance but to enhance NIV efficiency. However, a degree of mask leak is acceptable and the levels of leakage recorded across both cohorts was low overall therefore was not likely to be clinical significant.

Children with DS are more susceptible to developing the adverse sequelae of OSA largely due to the myriad of co-morbidities ${ }^{27}$, and as such The American Academy of Pediatrics (AAP) recommend a screening PSG for OSA be carried out before the age of 4 in all children with DS, a sentiment echoed by the Royal College of Paediatrics and Child Health ${ }^{36-38}$. Up to $60 \%$ of children with DS have a history of congenital and structural heart disease increasing their predisposition to persistent, development and recurrence of pulmonary hypertension if OSA goes untreated or undertreated ${ }^{14,15,26}$. Congenital heart disease has previously been suggested to undermine the efficacy of upper airway surgical outcome ${ }^{22}$. Those with a history of congenital heart disease and particularly those previously requiring cardiac surgery within the DS population have an 
increased risk of the cardiac sequelae of untreated OSA.

In this study, seventy-seven percent of this DS cohort has a past medical history that includes cardiac disease. This higher than normal percentage is likely due to the fact that our centre is the national referral centre for congenital heart disease. We have shown that those with a prior history of cardiac surgery have significantly poorer NIV adherence compared with their non-surgical counterparts.

To date, this is the first study of its kind that has identified poor NIV adherence within a DS cohort with prior cardiac surgery. Similarly, previous studies have noted poor therapeutic compliance within an adolescent and young adult cohort following heart or heart and lung transplant ${ }^{39}$. In this study by Wray et al, unintentional and intentional non-adherence was assessed, factors surrounding intentional non-compliance included perceived difficulty of the therapeutic intervention, as well as, perceived benefit ${ }^{39}$. Treatment compliance in long-term paediatric conditions is a complex issue, it must be looked at in the context of the issue at hand, past medical and surgical history of the child as well as the interaction of the family with healthcare and practitioners ${ }^{40}$. Parental non-compliance commonly arises from the emotional stresses associated with the recommended treatment; NIV initiation can be difficult and emotionally taxing on caregivers. Parents make continued attempts to balance concerns about the treatment, such as perceived effectiveness and the perceived long-term threat of the condition ${ }^{41}$. Impaired quality of life, as well as psychosocial issues including, adolescence, racial/ethnic minority status, and presence of mental health issues have been identified as factors associated with non-compliance ${ }^{42}$. Familial predictors associated with nonadherence problems included single-parent households, lower socioeconomic status, lower family cohesion, presence of family conflict, and poor family communication ${ }^{42}$. It could be postulated that the perceived threat of OSA is not as great as congenital heart disease and the therapeutic intervention is viewed as labour intensive particularly in the context of milder threat. These data highlight that increased surveillance and follow-up may be required in children with DS on NIV who have undergone prior cardiac surgery.

The major strength of this study is the inclusion of a control group. Previous similar studies have reported only on DS cohorts and the addition of a non-DS cohort adds significant weight to our findings. The authors are aware of the limitations of this study. Firstly, this is a single-centre retrospective study. The foremost scientific limitation of this study was the restricted availability of follow-up PSG data once NIV had been initiated, PSG data was not available to support and compliment the at home recordings at the investigated time-points due to limited resources. However the use of downloadable data from these NIV machines is emerging across the literature demonstrating its importance within clinical decision-making ${ }^{32,43}$. Home NIV data is becoming a key source of reliable information; it is not subject to the recall bias of previous usage questionnaires and can inform the clinician while helping to optimise patient care in a personalised fashion 32,43 .

In conclusion, this study confirms satisfactory NIV adherence in children with DS compared with a nonDS population. Particular attention should be given to children with DS and co-existing congenital heart disease in respect to NIV adherence. As we enter an age of personalised medicine, future studies should examine ways of improving NIV adherence and efficacy further in children with personalised NIV masks that incorporate 3D solutions to reduce system leak and discomfort ${ }^{44}$. Our study demonstrates that NIV delivery in complex children in a tertiary paediatric setting can be successful and that good adherence and efficacy levels can be achieved.

\section{Conflict of interest}

The authors declare no conflicts of interest.

\section{References}

1. Antonarakis SE, Lyle R, Dermitzakis ET, Reymond A, Deutsch S. Chromosome 21 and down syndrome: from genomics to pathophysiology. Nat Rev Genet. 2004;5(10):725-738.

2. Loane M, Morris JK, Addor MC, et al. Twenty-year trends in the prevalence of Down syndrome and other trisomies in Europe: impact of maternal age and prenatal screening. Eur J Hum Genet.2013;21(1):27-33. 
3. de Groot-van der Mooren MD, Tamminga S, Oepkes D, Weijerman ME, Cornel MC. Older mothers and increased impact of prenatal screening: stable livebirth prevalence of trisomy 21 in the Netherlands for the period 2000-2013. Eur J Hum Genet. 2018;26(2):157-165.

4. Rodman R, Pine HS. The otolaryngologist's approach to the patient with Down syndrome. Otolaryngol Clin North Am.2012;45(3):599-629, vii-viii.

5. Hamilton J, Yaneza MM, Clement WA, Kubba H. The prevalence of airway problems in children with Down's syndrome. Int J Pediatr Otorhinolaryngol. 2016;81:1-4.

6. Stoll C, Dott B, Alembik Y, Roth MP. Associated congenital anomalies among cases with Down syndrome. Eur J Med Genet.2015;58(12):674-680.

7. Maris M, Verhulst S, Wojciechowski M, Van de Heyning P, Boudewyns A. Prevalence of Obstructive Sleep Apnea in Children with Down Syndrome.Sleep. 2016;39(3):699-704.

8. Lee CF, Lee CH, Hsueh WY, Lin MT, Kang KT. Prevalence of Obstructive Sleep Apnea in Children With Down Syndrome: A Meta-Analysis. J Clin Sleep Med. 2018;14(5):867-875.

9. Beckhaus AA, Castro-Rodriguez JA. Down Syndrome and the Risk of Severe RSV Infection: A Metaanalysis. Pediatrics. 2018;142(3).

10. Ram G, Chinen J. Infections and immunodeficiency in Down syndrome. Clin Exp Immunol. 2011;164(1):916.

11. Galleguillos C, Galleguillos B, Larios G, Menchaca G, Bont L, Castro-Rodriguez JA. Down's syndrome is a risk factor for severe lower respiratory tract infection due to respiratory syncytial virus. Acta Paediatr. 2016;105(11):e531-e535.

12. Goffinski A, Stanley MA, Shepherd N, et al. Obstructive sleep apnea in young infants with Down syndrome evaluated in a Down syndrome specialty clinic. Am J Med Genet A. 2015;167A(2):324-330.

13. Chamseddin BH, Johnson RF, Mitchell RB. Obstructive Sleep Apnea in Children with Down Syndrome: Demographic, Clinical, and Polysomnographic Features. Otolaryngol Head Neck Surg. 2019;160(1):150-157.

14. Pandit C, Fitzgerald DA. Respiratory problems in children with Down syndrome. J Paediatr Child Health. 2012;48(3):E147-152.

15. Kaditis AG. Management of sleep-disordered breathing in children with complex disorders: One size does not fit all. Pediatr Pulmonol. 2018;53(10):1333-1335.

16. Alsubie HS, Rosen D. The evaluation and management of respiratory disease in children with Down syndrome (DS). Paediatr Respir Rev.2018;26:49-54.

17. Horne RS, Wijayaratne P, Nixon GM, Walter LM. Sleep and sleep disordered breathing in children with down syndrome: Effects on behaviour, neurocognition and the cardiovascular system. Sleep Med Rev. 2019;44:1-11.

18. Marcus CL, Moore RH, Rosen CL, et al. A randomized trial of adenotonsillectomy for childhood sleep apnea. N Engl J Med.2013;368(25):2366-2376.

19. Best J, Mutchnick S, Ida J, Billings KR. Trends in management of obstructive sleep apnea in pediatric patients with Down syndrome.Int J Pediatr Otorhinolaryngol. 2018;110:1-5.

20. Dudoignon B, Amaddeo A, Frapin A, et al. Obstructive sleep apnea in Down syndrome: Benefits of surgery and noninvasive respiratory support.Am J Med Genet A. 2017;173(8):2074-2080.

21. Farhood Z, Isley JW, Ong AA, et al. Adenotonsillectomy outcomes in patients with Down syndrome and obstructive sleep apnea.Laryngoscope. 2017;127(6):1465-1470. 
22. da Rocha M, Ferraz RCM, Guo Chen V, Antonio Moreira G, Raimundo Fujita R. Clinical variables determining the success of adenotonsillectomy in children with Down syndrome. Int J Pediatr Otorhinolaryngol. 2017;102:148-153.

23. Trucco F, Chatwin M, Semple T, Rosenthal M, Bush A, Tan HL. Sleep disordered breathing and ventilatory support in children with Down syndrome. Pediatr Pulmonol. 2018;53(10):1414-1421.

24. Simpson R, Oyekan AA, Ehsan Z, Ingram DG. Obstructive sleep apnea in patients with Down syndrome: current perspectives. Nat Sci Sleep.2018;10:287-293.

25. Trois MS, Capone GT, Lutz JA, et al. Obstructive sleep apnea in adults with Down syndrome. J Clin Sleep Med. 2009;5(4):317-323.

26. Hawkins A, Langton-Hewer S, Henderson J, Tulloh RM. Management of pulmonary hypertension in Down syndrome. Eur J Pediatr.2011;170(7):915-921.

27. Maris M, Verhulst S, Wojciechowski M, Van de Heyning P, Boudewyns A. Sleep problems and obstructive sleep apnea in children with down syndrome, an overwiew. Int J Pediatr Otorhinolaryngol.2016;82:12-15.

28. Berry RB, Budhiraja R, Gottlieb DJ, et al. Rules for scoring respiratory events in sleep: update of the 2007 AASM Manual for the Scoring of Sleep and Associated Events. Deliberations of the Sleep Apnea Definitions Task Force of the American Academy of Sleep Medicine.J Clin Sleep Med. 2012;8(5):597-619.

29. Berry RB, Gamaldo CE, Harding SM, et al. AASM Scoring Manual Version 2.2 Updates: New Chapters for Scoring Infant Sleep Staging and Home Sleep Apnea Testing. J Clin Sleep Med. 2015;11(11):1253-1254.

30. Farrell L, Cox DW. Outpatient initiation of non-invasive ventilation in children. Pediatr Pulmonol. 2019;54(1):10.

31. Brooks LJ, Olsen MN, Bacevice AM, Beebe A, Konstantinopoulou S, Taylor HG. Relationship between sleep, sleep apnea, and neuropsychological function in children with Down syndrome. Sleep Breath. 2015;19(1):197-204.

32. Mansell SK, Cutts S, Hackney I, et al. Using domiciliary non-invasive ventilator data downloads to inform clinical decision-making to optimise ventilation delivery and patient compliance.BMJ Open Respir Res. 2018;5(1):e000238.

33. Zhu K, Rabec C, Gonzalez-Bermejo J, et al. Combined effects of leaks, respiratory system properties and upper airway patency on the performance of home ventilators: a bench study. BMC Pulm Med.2017;17(1):145.

34. Piper AJ. Advances in non-invasive positive airway pressure technology. Respirology. 2019.

35. Maris M, Verhulst S, Wojciechowski M, Van de Heyning P, Boudewyns A. Outcome of adenotonsillectomy in children with Down syndrome and obstructive sleep apnoea. Arch Dis Child. 2017;102(4):331-336.

36. Sanders E, Hill CM, Evans HJ, Tuffrey C. The Development of a Screening Questionnaire for Obstructive Sleep Apnea in Children with Down Syndrome. Front Psychiatry. 2015;6:147.

37. Lagan N, Huggard D, Mc Grane F, et al. Multiorgan involvement and management in children with Down Syndrome. Acta Paediatr. 2020.

38. Bull MJ, Committee on G. Health supervision for children with Down syndrome. Pediatrics. 2011;128(2):393-406.

39. Wray J, Waters S, Radley-Smith R, Sensky T. Adherence in adolescents and young adults following heart or heart-lung transplantation.Pediatr Transplant. 2006;10(6):694-700.

40. Menahem S, Halasz G. Parental non-compliance-a paediatric dilemma. A medical and psychodynamic perspective. Child Care Health Dev. 2000;26(1):61-72. 
41. Santer M, Ring N, Yardley L, Geraghty AW, Wyke S. Treatment non-adherence in pediatric long-term medical conditions: systematic review and synthesis of qualitative studies of caregivers' views. BMC Pediatr. 2014;14:63.

42. Killian MO, Schuman DL, Mayersohn GS, Triplett KN. Psychosocial predictors of medication nonadherence in pediatric organ transplantation: A systematic review. Pediatr Transplant.2018;22(4):e13188.

43. Machaalani R, Evans CA, Waters KA. Objective adherence to positive airway pressure therapy in an Australian paediatric cohort. Sleep Breath. 2016;20(4):1327-1336.

44. Shikama M, Nakagami G, Noguchi H, Mori T, Sanada H. Development of Personalized Fitting Device With 3-Dimensional Solution for Prevention of NIV Oronasal Mask-Related Pressure Ulcers. Respir Care.2018;63(8):1024-1032.

\section{Hosted file}

Table 1 LMD.pdf available at https://authorea.com/users/362538/articles/483654-efficacy-andadherence-of-non-invasive-ventilation-treatment-in-children-with-down-syndrome
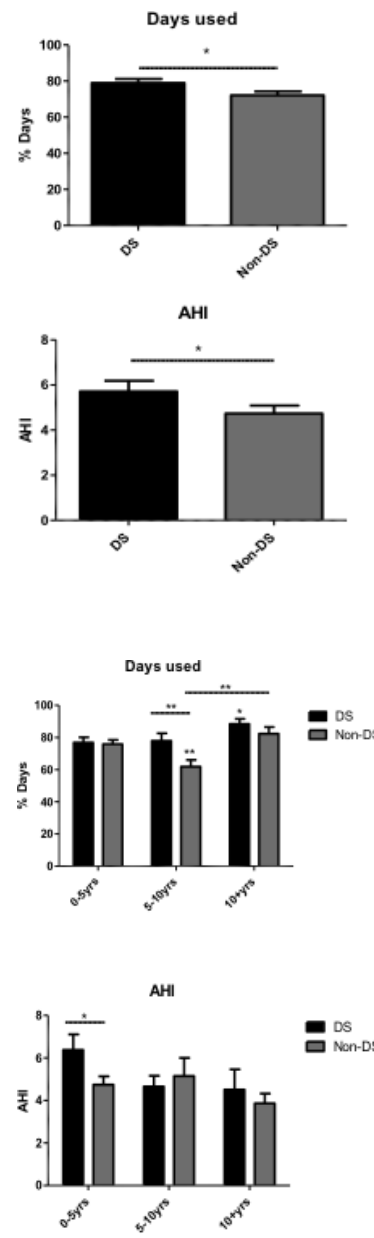
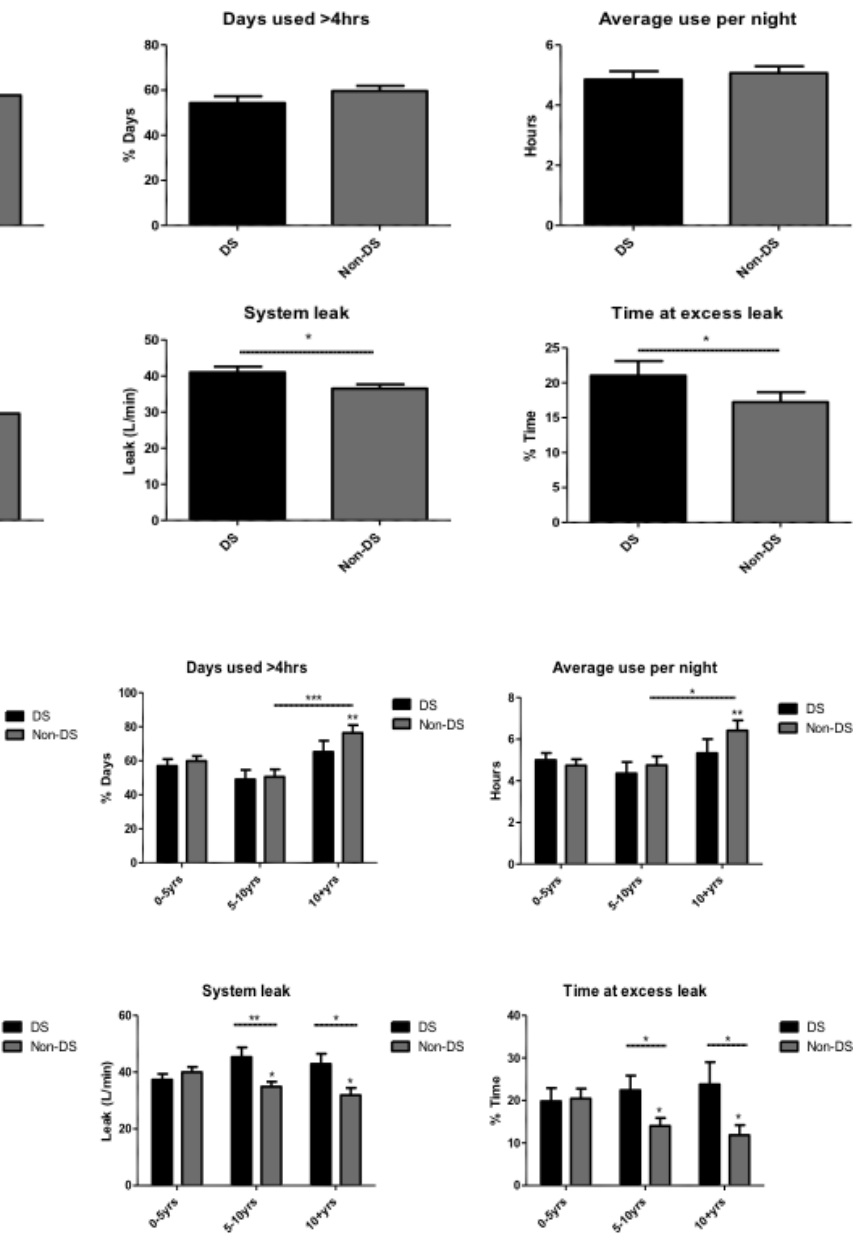

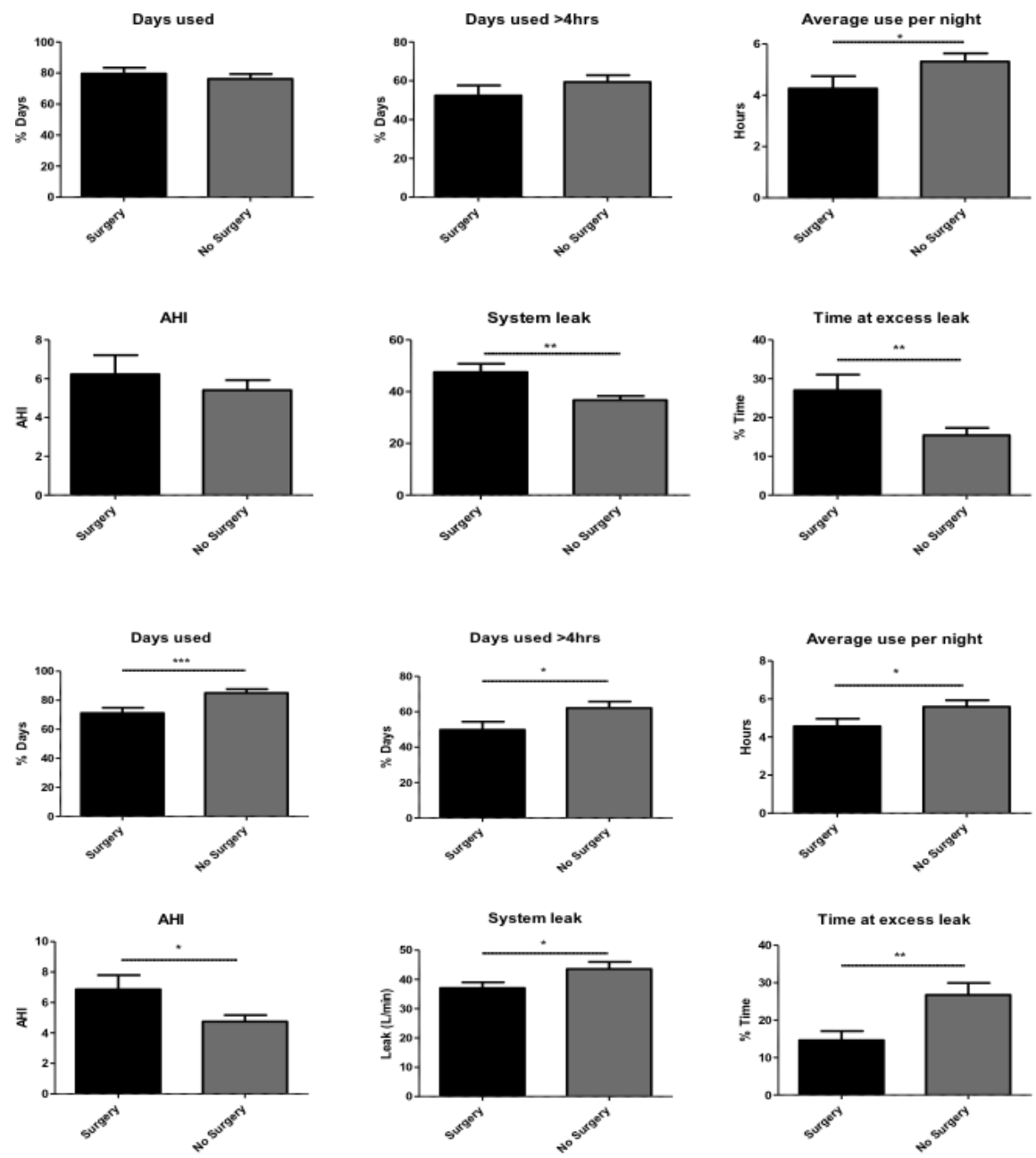